\title{
Furnace Chemical Vapor Deposition (FCVD) method for special optical fibers fabrication
}

\author{
A.A. Malinin*a , A.S. Zlenko ${ }^{\text {b }}$, U.G. Akhmetshin ${ }^{\text {c }}$, S.L. Semjonov ${ }^{\text {b }}$ \\ ${ }^{a}$ Optogear Oy, Kuormatie 14, Nummela, FI-03100, Finland \\ ${ }^{\mathrm{b}}$ Fiber Optics Research Center, Russian Academy of Sciences, 38 Vavilov st., 119333 Moscow, \\ Russia; \\ ${ }^{\mathrm{c}}$ Aston University, Aston Triangle 423, North Wing, Photonics Research Group, B4 7ET UK
}

\begin{abstract}
The features of the Furnace Chemical Vapor Deposition (FCVD) method of manufacturing preforms for special optical fibers are considered. It is shown that misalignment of substrate silica tube and furnace hole axes has a negative effect on the quality of fabricated preforms, leading to angular and radial asymmetry of the refractive index profile. Ways of getting rid of this and other disadvantages of the FCVD method are described. Some advantages of the FCVD method over the MCVD method are shown. It was demonstrated that the FCVD method, despite some drawbacks, allows to manufacture high-quality fiber preforms with good symmetry of the refractive index profile, and thus it is promising for fabrication of dispersion, dispersion varying and active fibers.
\end{abstract}

Keywords: special fiber, preform fabrication, FCVD, MCVD, dispersion, RIP, symmetry, fiber technology.

\section{INTRODUCTION}

The quality of an optical fiber directly depends on the quality of initial preform. To date there is a lot of technological processes for optical fiber preform manufacturing: OVD, VAD, APVD, MCVD, FCVD and PCVD [1]. The latter three methods are very similar. We consider the main features of the FCVD method (Furnace Chemical Vapor Deposition) [2]. In spite of the fact that the FCVD method has certain advantages and was used, in particular, by firms Draka and Alcatel, there are few publications on this method and references in the majority of cases have indirect character [3].

\section{FCVD AND MCVD COMMON FEATURES}

As well as in the MCVD method glass layers are formed by thermophoretic deposition of oxidation products of the initial reagents $\left(\mathrm{SiCl}_{4}, \mathrm{GeCl}_{4}, \mathrm{POCl}_{3}, \mathrm{BBr}_{3}\right.$, etc) in a substrate silica tube at high temperature and their subsequent fusing.

The FCVD and MCVD methods share the following advantages and disadvantages.

Advantages:

1. Relative simplicity of the machine tool design;

2. The formation of layers is going on inside the silica tube, therefore it is easy to provide isolation of the reaction zone from external contaminants;

3. It is possible to manufacture the refractive index profile (RIP) with good accuracy, since the methods allow to control efficiently the deposition of layers.

Disadvantages:

1. The high-purity silica glass tubes with good geometric parameters are required;

2. Diffusion of impurity from the substrate tube to the preform core is possible;

3. The length of preform is limited by machine tool dimensions, hence preforms have a small size;

*alexei.malinin@optogear.fi; phone+358 9222 7799; fax +358 222 7789; optogear.fi 
4. The central dip in the distribution of RIP is possible, because the tube collapsing process requires heating up to the silica glass softening temperature, and as a result some dopants (e.g. oxides of germanium or phosphorus) evaporate from the inner surface of the tube;

5. Low speed of glass formation: 0.5-2.0 grams per minute;

6. It is necessary to take into account the change of temperature inside the tube depending on the number of deposited layers;

7. Variation of the RIP along the preform is possible.

\section{FCVD FEATURES}

Despite the FCVD method has numerous characteristics common with those of the MCVD, it has some features of its own, making it promising for the special optical fibers manufacturing [4].The main difference of the FCVD method from the MCVD one is that the oxygen - hydrogen burner is replaced by an electric furnace (Fig. 1a). The replacement of a burner with the furnace eliminates a number of disadvantages, such as the nonuniformity of thermal field in a heating zone, the diffusion of hydrogen from the torch flame into preform, the strong evaporation of silica from the external surface of the tube. It should be noted that the nonuniform thermal field in the heating zone is often the cause of serious defects in the preform, for example, its ovality or the angular inhomogeneity of the refractive index profile. Accordingly, it is rather difficult to obtain from such preform the fiber with good characteristics. The main advantage of FCVD consists in the fact that uniform heating from all the sides provides optimal conditions for fabrication of symmetrical preforms, both in the form and RIP distribution. In other words, the formation of the layers, and the collapsing process are going on uniformly if the tube has no geometrical defects.

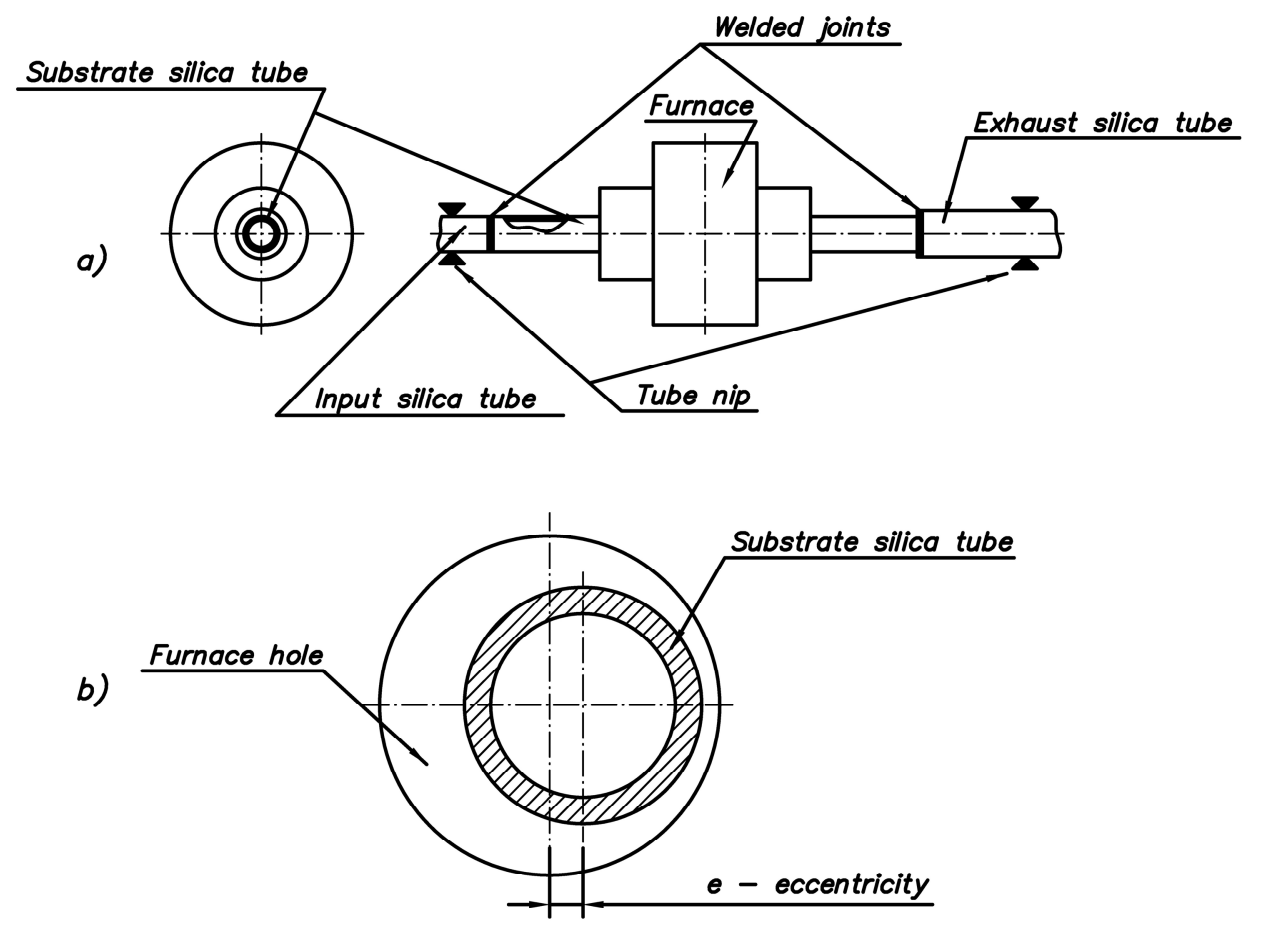

Fig. 1 The substrate silica tube positioning relative to the furnace in the FCVD method.

The FCVD method has some disadvantages as well. The RIP symmetry is highly dependent on the coaxiality of furnace hole and substrate tube hole. In the case of presence eccentricity $\boldsymbol{e}$ (Fig. 1b), the RIP symmetry becomes worse because one side of substrate tube all the time is closer to the heating element and temperature field becomes nonuniform on the tube external surface. Due to the non-uniform temperature field the degree of doping varies around the 
circumference, as the incorporation of impurities depending on the temperature occurs differently. And therefore the refractive index also varies around the circumference.

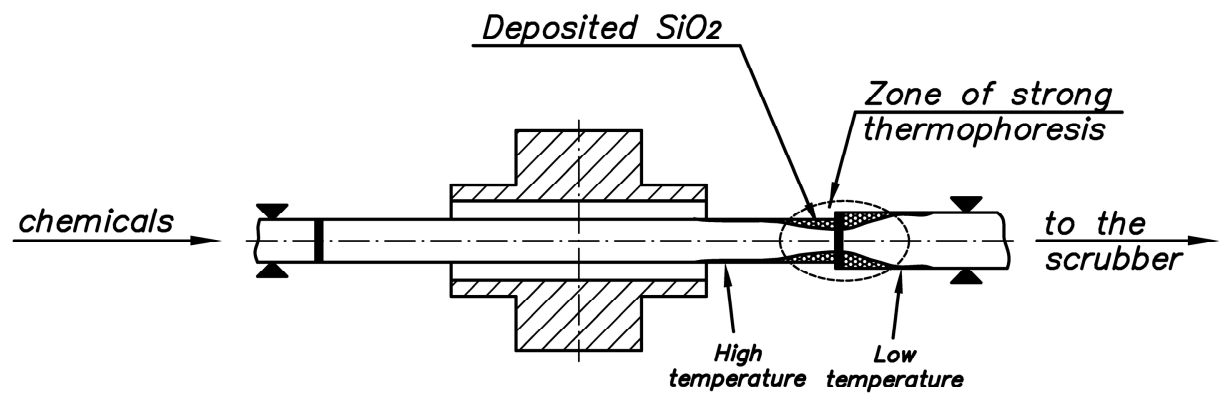

Fig. 2. Intensive thermophoresis of silicon dioxide near the weld between the substrate and exhaust tubes leads to a narrowing of the internal diameter of the tube, and even to the tube clogging

In the process of preform manufacturing an "input tube - substrate tube - exhaust tube" welded construction is usually used (Fig. 1). It allows the substrate tube to be aligned at the initial stage of the manufacturing process with a high degree of flexibility. Input silica tube is rigidly connected to the chemical feed system. Usage of rigidly fixed tube as an input tube improves sealing to prevent ingress of impurities, and also reduces the time spent on aligning the tubes. The exhaust silica tube is always taken of a larger diameter than that of the substrate tube, because it is need to reduce the intensity of silicon dioxide deposition inside the substrate tube near the place where the direction of the furnace movement is changed (usually near the weld of the substrate and exhaust tubes). Intensive silicon dioxide deposition occurs due to the strong thermophoresis because of large temperature gradient near this place (Fig. 2). Due to a larger diameter of exhaust tube the most part of white soot deposited near the end of the furnace pass is blown out into the scrubber.

The increased coaxiality requirements impose additional restrictions on the quality of welds between the substrate tube and the input and exhaust tubes, on the geometrical parameters of tubes, on the quality of tube chucking devices. In the process of fiber preform manufacturing, an eccentricity may arise as a result of the silica tube sagging. The tube sagging may occur owing to the influence of the following reasons:

1) For the oxidation reaction to occur, the rotating tube is heated to the silica glass softening temperature, this results in axis vibrations in the heating zone due to gravity (Fig. 3a).

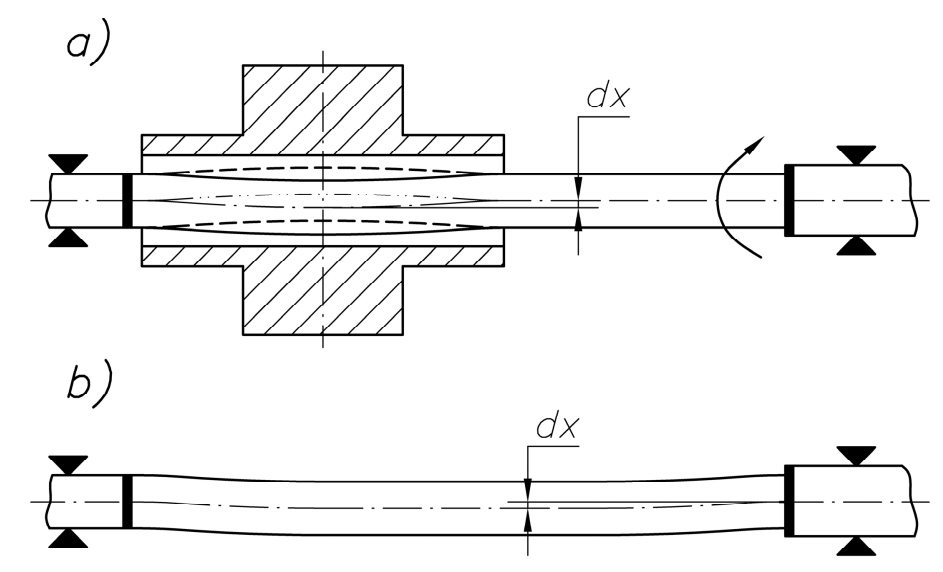

Fig. 3. Schematic of a. Axis vibrations in the heating zone due to gravity, b. Tube sagging, due to gravity after one furnace pass.

Ideally, the rotation should prevent the tube sagging in the heating zone, but in practice the tube may cool down with locally displaced axis. As a result of the furnace pass, the tube may sag as shown in Fig. 3b. This usually occurs when a misalignment of the axes $\mathrm{f}$ the tubes exists already. 


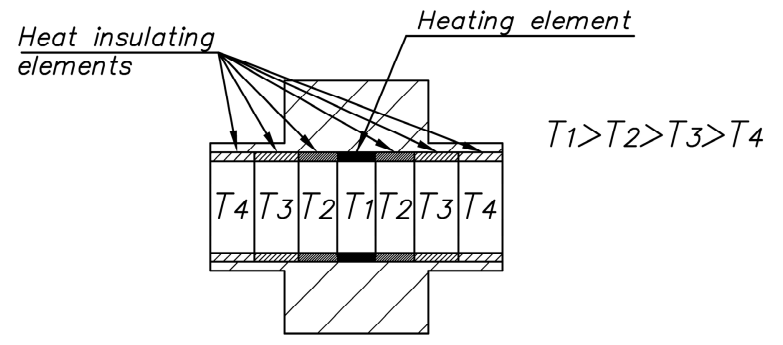

Fig. 4. Heating temperature varies along the length of the furnace. The maximum temperature is reached in the furnace center - in the region of the graphite heating element. The temperature decreases with the distance from the center.

Such sagging is stimulated by a wide heating zone of the furnaces (Fig. 3a, 4) and a presence of mechanical stresses in the silica tubes.

2) It is necessary to heat by the furnace the welded joint of the substrate silica tube and the exhaust silica tube for thermophoresis reducing near this zone (Fig. 2). Heating of welded joint to the silica softening temperature leads to more intensive sagging of the tube (Fig. 5)

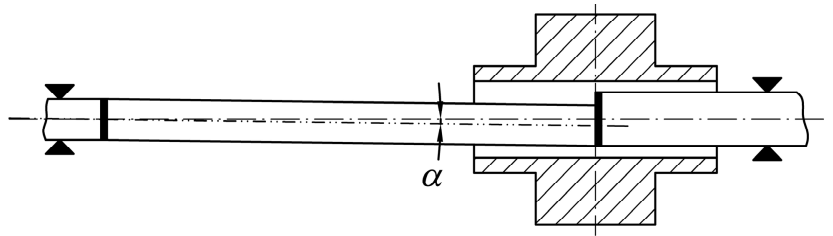

Fig. 5. Deflection of a tube under the influence of gravity as a result of welded joint heating.

This problem is caused by heating features of the furnace. The graphite heating element is surrounded by a heat insulators, which reduces the temperature closer to the furnace edges, but they are also heated strongly enough. As a result the furnace has rather wide heating zone with a maximum temperature located at the center, where the heating element is installed (Fig. 4). These features of the heating zone lead to the fact that, if not to bring the furnace center up to the weld, the temperature gradient near the joint of the tubes will be too much, and even the usage of exhaust tube and exhaust ventilation will not allow to avoid the tube clogging by silicon dioxide (Fig. 6a). In the MCVD method, this problem is negligible, because the burner has a much smaller size and approximately uniform heating zone. So it is possible to lead up the torch to the end of a substrate tube (Fig. 6). And the zone of strong thermophoresis will be in an exhaust tube and will not really affect the weld. Deposited in this zone silicon dioxide with the help of exhaust ventilation will almost completely be blown out into the scrubber.

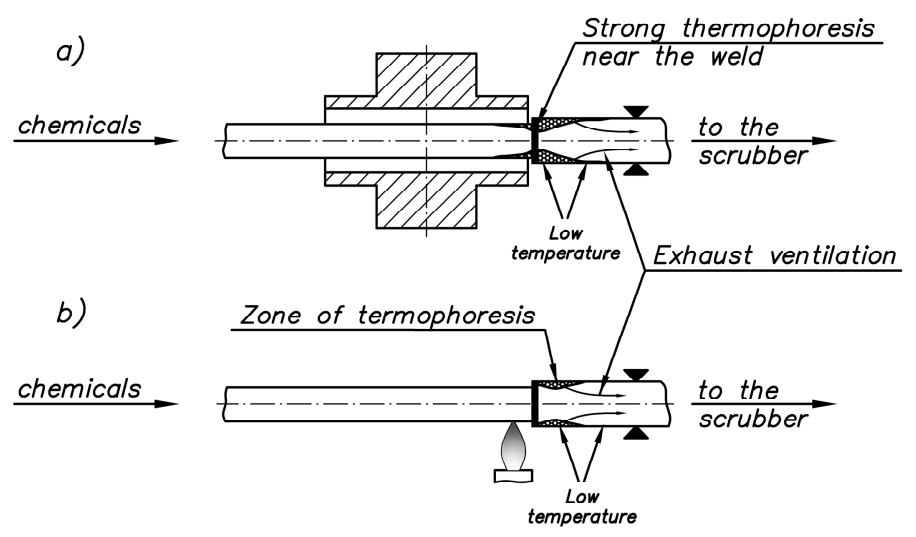

Fig. 6. Comparison of silicon dioxide deposition near the substrate and exhaust tube weld in FCVD (a) and MCVD (b) 


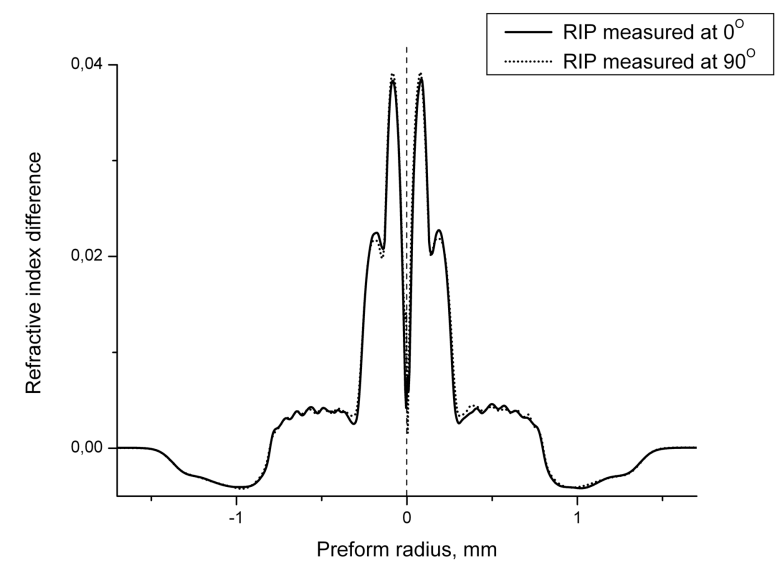

Fig. 7. Example of fiber preform RIP with good symmetry

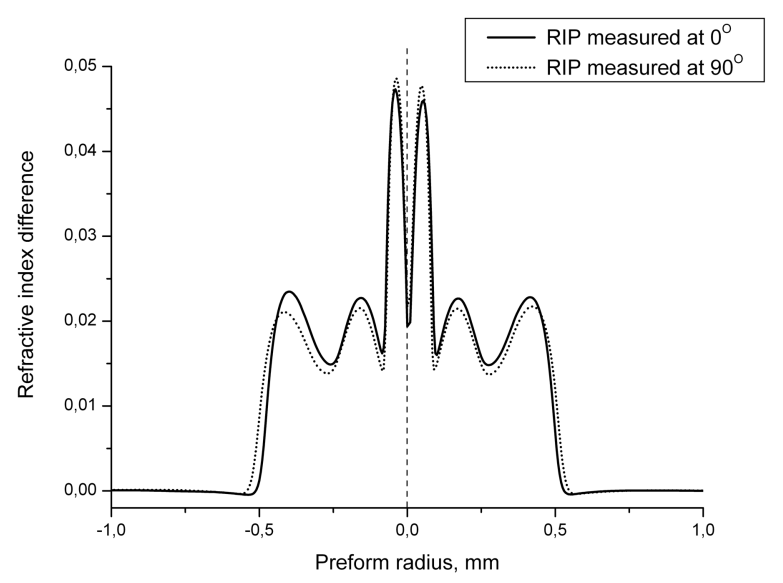

Fig. 8. Example of fiber preform RIP with symmetry not as good as in Fig. 7.

Fig. 7 shows RIP of the preform with good angular and radial symmetry (refractive index profiles were measured by York Technology P102 Preform Analyzer) because of the small axis misalignment of silica tube and furnace hole, and Fig. 8 shows RIP of the preform, in the manufacturing process of which larger axis misalignment (as shown in Fig.1b) has arisen. The symmetry of the RIP in Fig. 8 is worse than in Fig. 7, but nevertheless fairly good.

Besides, the furnace has a large mass, which requires time for a smooth stop/start of motion (if to stop abruptly, the mechanism of furnace movement can be damaged), this leads to uneven tube heating and therefore has negative effect on the identity of the RIP along the preform length (especially at the preform ends).

At the same time, influences of all mentioned disadvantages on the preform quality can be minimized by means of total control and optimization of parameters of the preform manufacturing process. In particular, in order to avoid the formation of large eccentricity, it is necessary to find the optimal length of utilized tubes. It should be taking into account that this length may vary for different conditions depending on composition, diameter and thickness of the tube, and also width of a heating element of the furnace. Irregularity of profile along the preform length can be reduced by optimizing of reagents flows through the tube, velocity and temperature of the furnace for each preform layer (for each pass of furnace).

\section{FCVD FOR SPECIAL OPTICAL FIBERS MANUFACTURE}

Special optical fibers have found wide application in numerous areas of science and technology. For example, in nonlinear optics special fibers with small mode field diameter [5] or with non-standard dispersion are widely used [6,7]. Observance of rather strict requirements on the symmetry of the radial refractive index distribution is necessary for creation of such fibers, because even insignificant changes or asymmetry of the refractive index profile may negatively 
affect the optical characteristics of fiber (such as chromatic dispersion, mode field diameter, effective nonlinear refractive index, etc) [8].

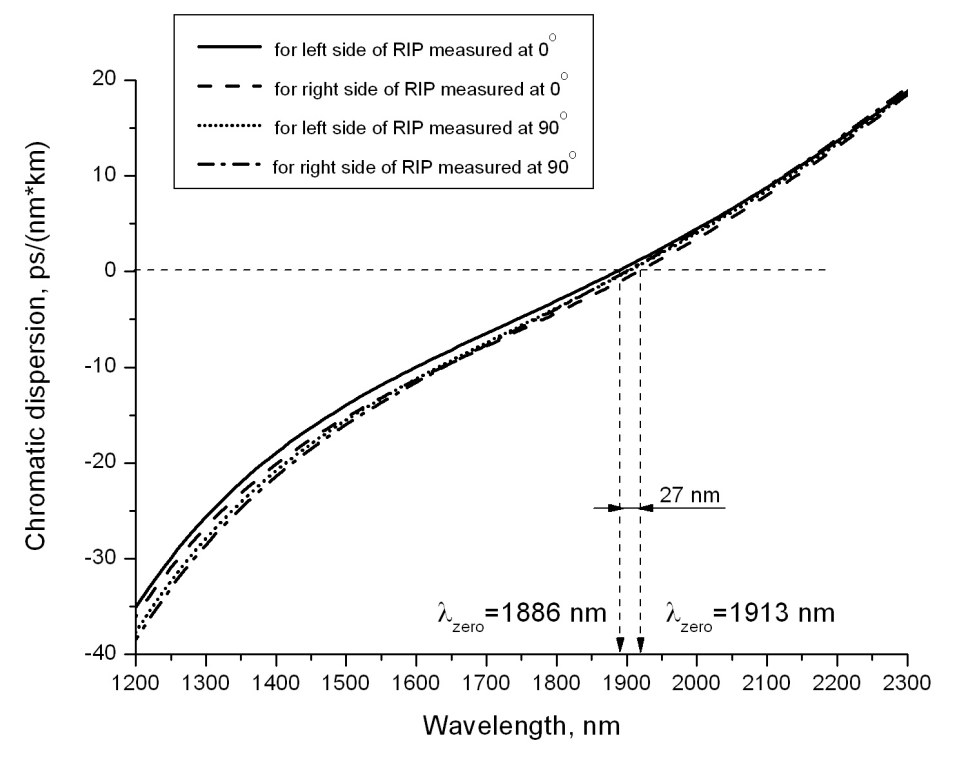

Fig. 9. Chromatic dispersion calculated for left and right side of RIP in Fig. 7.

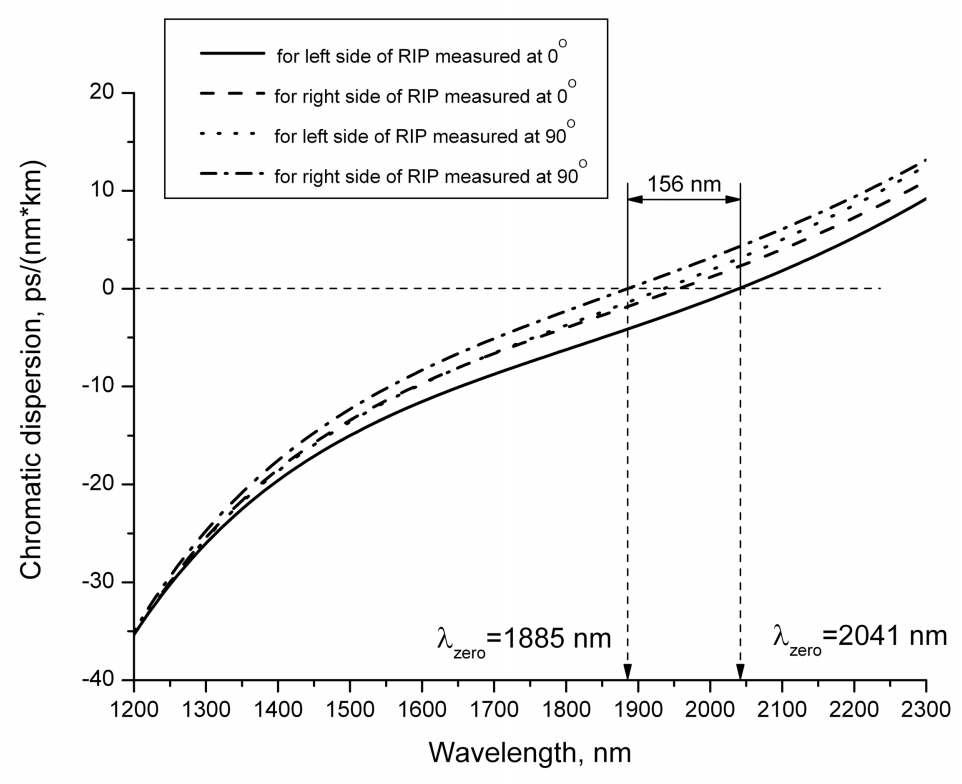

Fig. 10. Chromatic dispersion calculated for left and right side of RIP in Fig. 8.

As an example of the high sensitivity of the fiber optical properties to the RIP symmetry, figures 9 and 10 show the calculated spectral dependence of chromatic dispersion for left and right halves of the refractive index profiles in Fig. 7 and 8. As seen from this figures dispersion varies if RIP has asymmetry. But if for a profile with good symmetry (Fig. 7) this variation is small (Fig. 9) for a profile on Fig. 8 it is more significant (Fig. 10), so the zero dispersion wavelength spread is $156 \mathrm{~nm}$. The real value of dispersion in optical fibers, drawing from these preforms will be placed between two 
extreme dispersion curves. And it may be approximately estimated for each wavelength as an average between calculated values of dispersion on this wavelength for different RIP halves. Thus, the asymmetry of the RIP may be an obstacle for creation of dispersion optical fibers, as because of asymmetry it is difficult to abide the dispersion curve with a given accuracy [8]. Besides, the angular asymmetry of the profile may lead to an increase in polarization mode dispersion and angular scattering that is also undesirable in many applications. Symmetry of the preform in Fig. 7 was achieved, owing to reduction of substrate tube length. But as seen in Fig.9, even such, almost full symmetry is not enough and there are divergences in the optical parameters calculated for RIP, measured in different planes. Since the small angular asymmetry of RIP is inevitable, then besides minimizing of axes misalignment, it is necessary to use such RIP structures for which the optical parameters change insignificantly with small variations in the refractive index [8].

\section{CONCLUSIONS}

Research have shown that the FCVD method have rich potential for special optical fibers manufacturing. Preforms fabricated by FCVD have good symmetry of RIP therefore the FCVD method is especially attractive for dispersion, varying along a length and active fibers manufacturing [9]. Optical fibers made from such preforms have good characteristics and, hence, may find wide application in various fields of fiber optics.

\section{REFERENCES}

[1] Cognolato, L., "Chemical Vapour Deposition for Optical Fibre Technology", Proceedings of the Tenth European Conference on Chemical Vapour Deposition, J. Phys. IV France 05, C5-975-C5-987 (1995)

[2] Le Noane, G., Hardy, I., and Grosso, P., "Preform manufacturing by FCVD", IWCS 93, St Louis, USA, 1993.

[3] Bethuys, S., Lablonde, L., Rivoallan, L. and Auvray, P., " $1 \times 4$ fused multicore singlemode fibre coupler: theory, fabrication and analysis", Electron. Lett. 34, 1516-1517 (1998)

[4] Zlenko, A.S., Akhmetshin, U.G., "Features of FCVD method of manufacturing fiber preforms for use in nonlinear optics and laser applications", The third Russian seminar on fiber lasers, pp. 33-35, Ufa, 2009 (in Russian).

[5] Onishi, M., New nonlinear fibers with application to amplifiers, in: Proceedings of the Optical Fiber Communication Conference (OFC), TuC3, Los Angeles, 2004.

[6] Zlenko, A.S., Bogatyrjov, V.A., Akhmetshin, U.G., Andrianov, A.V., Muraviev, S.V., "Optical fiber for research of nonlinear effects near 1,85 $\mu \mathrm{m}$ ", The third Russian seminar on fiber lasers, pp. 46-48, Ufa, 2009 (in Russian).

[7] Andrianov, A.V., Kim, A.V., Muraviev, S.V. and Sysoliatin, A.A., "DDF-based All-fiber Optical Source of Femtosecond Pulses Smoothly Tuned in the Telecommunication Range", Laser Physics 17, 1296-1302 (2007).

[8] Akhmetshin, U.G., "The research and development of the single-mode optical fibers with chromatic dispersion varying along the length", Ph.D. Thesis, A.M. Prokhorov General Physics Institute, Moscow, Russian Academy of Sciences, 2003 (in Russian).

[9] Zlenko, A.S., Akhmetshin, U.G., "Optical fiber doped with active nanofilms into the cladding. Method of preform manufacturing", The third Russian seminar on fiber lasers, pp. 44-46, Ufa, 2009 (in Russian). 